\section{Adaptación transcultural y validación de un instrumento de calidad de vida relacionada con la salud en adolescentes chilenos}

\author{
RODRIGO SEPÚLVEDA P. ${ }^{1, a}$, TEMÍSTOCLES MOLINA G. ${ }^{2, b}$, \\ RAMIRO MOLINA C. ${ }^{2}$, VANIA MARTÍNEZ N. ${ }^{2}$, \\ ELECTRA GONZÁLEZ A. 2,c , MYRIAM GEORGE L. ${ }^{3, \mathrm{~d}}$, \\ ROSA MONTAÑO E. .,e, CARLOS HIDALGO-RASMUSSEN ${ }^{5,6, \mathrm{f}}$
}

\section{Validation of an instrument to measure health-related quality of life in Chilean children and adolescents}

Background: KIDSCREEN-52 is an instrument to assess health related quality of life in children and adolescents. Aim: To culturally adapt and validate the KIDSCREEN-52 questionnaire in Chileans. Material and Methods: Two independent translations from the English Spanish language were conciliated and retranslated to English. The conciliated version was tested during a cognitive interview to adolescents of different socioeconomic levels. The final version was validated in 7,910 school attending adolescents. Results: In the cross-cultural adaptation, 50 of the 52 items presented low or medium levels of difficulty and a high semantic equivalence. Distribution according to gender, grades and types of schools was similar to the sample. Single ages were not affected by sex distribution. The Confirmatory Factor Analyses were: $\chi^{2}(1229)=20996.7$, Root Mean Square Error of Approximation $=.045$ and Comparative Fit Index $=.96$. The instrument had a Cronbach's alpha of .93. The domains had scores over 0.70 points, with the exception of the "Selfperception" domain, with a score of 0.62. Conclusions: The Chilean version of KIDSCREEN-52 is culturally appropriate and semantically equivalent in its English and Spanish versions (from Spain). Its reliability and validity were adequate.

(Rev Med Chile 2013; 141: 1283-1292).

Key words: Adaptation psychological; Adolescent; Cross-cultural Adaptation; quality of Life.

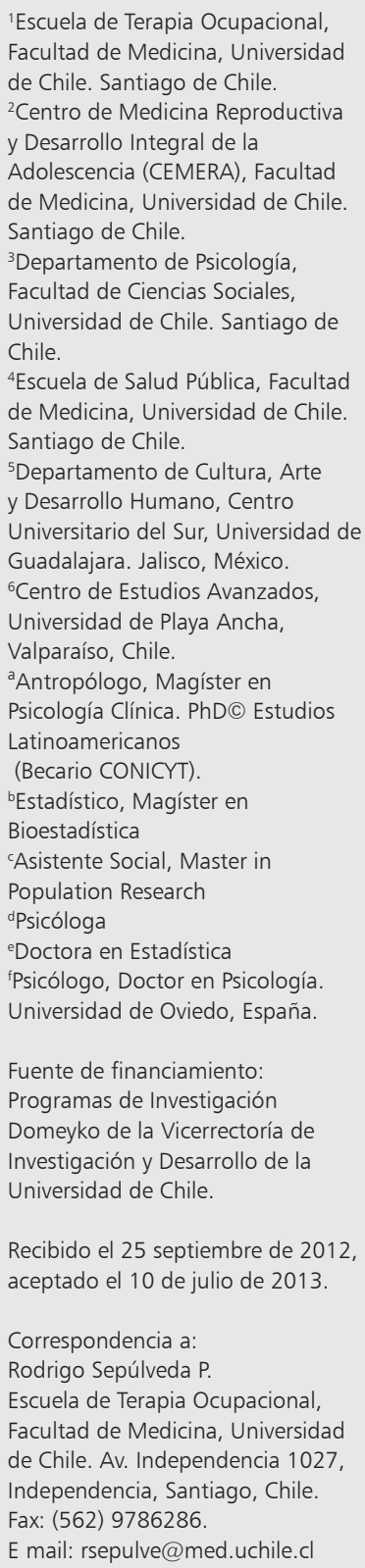

L os estudios de calidad de vida relacionada con la salud (CVRS), entendida como "la salud física y mental percibida individual o grupalmente en el tiempo"1, abarcan prácticamente todas las áreas de las especialidades en medicina ${ }^{2-4}$. Su uso es habitual en la evaluación de intervenciones quirúrgicas o en situaciones de patologías crónicas ${ }^{5,6}$. También se han aplicado en estudios de seguimiento en CVRS en niños y adolescentes ${ }^{7}$.

En Chile existen estudios de CVRS en adultos $^{8}$ y adolescentes ${ }^{9,10}$. Es necesario desarrollar instrumentos que permitan evaluar la CVRS en adolescentes para la formulación de políticas públicas e instalación de servicios y redes de atención en salud, ya que los estudios en CVRS describen 
variables trazadoras de la conducta de cuidado y autocuidado de la salud ${ }^{11}$.

El KIDSCREEN-52 mide CVRS en niños y adolescentes. Fue desarrollado en forma multicultural por un equipo de investigadores de la Universitäts klinikum Hamburg-Eppendorf de Alemania y colaboradores de distintos países europeos ${ }^{12,13}$. Este instrumento fue aplicado simultáneamente en 13 países europeos (Alemania, Austria, España, Francia, Grecia, Hungría, Irlanda, Países Bajos, Polonia y República Checa) ${ }^{12-15}$ y se ha adaptado y validado en países de habla hispana ${ }^{16-18}$. Tiene 10 dimensiones, con un total de 52 ítems, cuyo número se señala entre paréntesis: bienestar físico (5), bienestar psicológico (6), estado de ánimo y emociones (7), autopercepción (5), autonomía (5), relación con los padres y vida familiar (6), recursos económicos (3), amigos y apoyo social (6), entorno escolar (6) y aceptación social (bullying) (3). Los 52 ítems son respondidos en escala de Likert, con 5 niveles de respuesta.

La adaptación transcultural implica la evaluación de la equivalencia lingüística, conceptual y de las propiedades de medida ${ }^{18}$. El objetivo del presente estudio fue describir la adaptación cultural y evaluar la fiabilidad y la validez del cuestionario KIDSCREEN52 en Chile. De esta forma se espera contribuir con otros estudios nacionales y su comparabilidad internacional.

\section{Material y Método}

\section{Adaptación cultural}

De acuerdo con las guías sugeridas por el grupo KIDSCREEN ${ }^{19}$, el estudio se inició con la traducción del instrumento desde la versión original $^{20-22}$ en inglés al español y retrotraducción al inglés. En la traducción se utilizó como referencia otras versiones del instrumento en español, Chile (versión no adaptada transculturalmente) ${ }^{24}$ y España ${ }^{13}$. La versión de traducción reconciliada fue retrotraducida al inglés por una tercera traductora no relacionada a las anteriores traductoras, que residía en Chile y tenía al inglés como lengua materna. Esta última versión se expuso a entrevistas cognitivas con adolescentes, para asegurar la aceptabilidad y comprensión. Se seleccionaron 12 estudiantes en tres rangos de edad: 10-12, 1315 y 16-18 de ambos sexos, de 2 establecimientos educacionales de diferente nivel socio-económico (nivel socioeconómico alto y bajo). En cada rango se consideró 1 hombre y 1 mujer de modo que en ambos establecimientos se entrevistó 6 personas ( 3 mujeres y 3 hombres). Estas entrevistas cognitivas fueron aplicadas por una lingüista, especialista en análisis del discurso y una antropóloga, especializada en metodologías cualitativas que estaba familiarizada con el concepto de CVRS. Las entrevistas cognitivas se basaron en el método "paraphrasing", en el que se pide al o la adolescente que repita el ítem con sus propias palabras y el método "general probing" ${ }^{20}$, en el que se le pregunta si el ítem es considerado comprensible y claro. Todo el material fue audiograbado.

\section{Diseño muestral}

El instrumento adaptado y aprobado por el representante del KIDSCREEN Group, se validó verificando sus propiedades psicométricas ${ }^{25}$, para lo cual se aplicó el instrumento a una muestra de estudiantes a nivel nacional, provenientes de cursos entre quinto básico y cuarto medio (8 niveles), de establecimientos escolares pertenecientes a las tres modalidades enseñanza que existen en Chile (municipalizada, particular subvencionada y particular pagada).

El cálculo del tamaño muestral con significación nacional se basó en la información de la población escolar entre 10 y 18 años entregada por el Ministerio de Educación para $2007^{26}$. El criterio de selección se hizo a través de un muestreo aleatorio simple a partir de los resultados obtenidos del estudio CVRS realizado por el Ministerio de Salud en 2006 que obtuvo una prevalencia promedio de $\mathrm{p}=0,243$ para las variables consideradas pertinentes del presente estudio ${ }^{27}$.

La selección de alumnos a encuestar se hizo con un diseño probabilístico estratificado en dos etapas. La primera seleccionó los cursos de cada estrato y la segunda seleccionó los alumnos de cada curso elegido. La selección de los cursos se hizo con probabilidad proporcional según el número de alumnos por colegio y el número de cursos a seleccionar en el estrato. Así, los colegios con mayor número de alumnos tuvieron mayor probabilidad de selección y de esta forma en los colegios con mayor número de cursos se pudo seleccionar uno o más cursos por nivel. Considerando un nivel de confianza de $95 \%(Z=1,96)$ y el efecto del diseño al aplicar muestreo en dos etapas, se obtuvo 8.200 alumnos como muestra esperada que consideró un margen de pérdida de $10 \%$. 
Tabla 1. Características sociodemográficas de la población encuestada (n: 7.910)

\begin{tabular}{|c|c|c|c|c|c|c|c|c|c|c|c|c|}
\hline \multirow{2}{*}{\multicolumn{3}{|c|}{ Variables }} & \multicolumn{4}{|c|}{ Sexo } & \multicolumn{6}{|c|}{ Tipo de establecimiento escolar } \\
\hline & & & \multicolumn{2}{|c|}{ Mujer } & \multicolumn{2}{|c|}{ Hombre } & \multicolumn{2}{|c|}{ Municipal } & \multicolumn{2}{|c|}{ Subvencionado } & \multicolumn{2}{|c|}{ Pagado } \\
\hline Edad & $\mathbf{n}$ & $\%$ & $\mathbf{n}$ & $\%$ & $\mathbf{n}$ & $\%$ & $\mathbf{n}$ & $\%$ & $\mathbf{n}$ & $\%$ & $\mathbf{n}$ & $\%$ \\
\hline 10 & 477 & 6,0 & 292 & 3,7 & 185 & 2,3 & 163 & 2,1 & 196 & 2,5 & 118 & 1,5 \\
\hline 11 & 934 & 11,8 & 557 & 7,0 & 377 & 4,8 & 318 & 4,0 & 350 & 4,4 & 266 & 3,3 \\
\hline 12 & 1.028 & 13,0 & 622 & 7,9 & 406 & 5,1 & 370 & 4,7 & 384 & 4,9 & 274 & 3.4 \\
\hline 13 & 1.096 & 13,9 & 643 & 8,1 & 453 & 5,7 & 344 & 4,3 & 410 & 5,2 & 342 & 4,3 \\
\hline 14 & 982 & 12,4 & 489 & 6,2 & 493 & 6,2 & 311 & 3,9 & 363 & 4,6 & 308 & 3,9 \\
\hline 15 & 870 & 11,0 & 432 & 5,5 & 438 & 5,5 & 274 & 3,5 & 290 & 3,7 & 306 & 3,8 \\
\hline 16 & 1.050 & 13,27 & 468 & 5,9 & 582 & 7,4 & 340 & 4,3 & 357 & 4,5 & 353 & 4,5 \\
\hline 17 & 970 & 12,27 & 452 & 5,7 & 518 & 6,5 & 308 & 3,9 & 361 & 4,6 & 301 & 3,8 \\
\hline 18 & 503 & 6,36 & 237 & 3,0 & 266 & 3,4 & 149 & 1,9 & 207 & 2,6 & 147 & 1,9 \\
\hline Total & 7.910 & 100,0 & 4.192 & 53,0 & 3.718 & 47,0 & 2.577 & 32,6 & 2.918 & 37,0 & 2.415 & 30,4 \\
\hline
\end{tabular}

Se obtuvo 53\% de participantes de sexo femenino (Tabla 1), lo que estuvo en el promedio del estudio europeo original ${ }^{11,13}$. Se diseñó muestras comparativas para los tres tipos de educación para mantener las posibilidades de selección de ellas en todas las ciudades elegidas en cada zona. La muestra final fue de 7.910 por eliminación de las encuestas no respondidas, ausencia de alumnos el día de la encuesta, con menos de $25 \%$ de las preguntas respondidas ( 39 de 52 preguntas) $y$ edades fuera del rango definido en el estudio. La pérdida observada fue de 3,5\%, menor a $10 \%$ estimado. El trabajo de campo se realizó entre julio y octubre de 2009.

El diseño muestral según distribución geográfica se conforma por 24 estratos distribuidos en seis zonas de acuerdo a las 15 regiones de Chile: 2 en el norte, 2 en el centro incluyendo la Región Metropolitana y Costa, y 2 en el sur. La Tabla 2 recoge la muestra final obtenida de estudiantes por regiones y comunas. La proporción de alumnos seleccionados mantiene la distribución de la población escolar de Chile, con un peso relativo mayor en la Región Metropolitana y la de Valparaíso.

\section{Indicadores de validación utilizados}

\section{Puntajes Rasch}

Se utilizó el modelo teórico de crédito parcial (PCM) que pertenece a la familia de los modelos $\mathrm{RASCH}$, para calcular los puntajes Rasch para cada dimensión. Estas puntuaciones son posteriormente transformados a valores $\mathrm{T}$, correspondientes a una media de 50 y una desviación estándar de 10, para una mayor facilidad de uso y comparabilidad con los referentes españoles y europeos; mayores puntajes indican una mejor calidad de $\operatorname{vida}^{28}$. Se utilizó el programa WINSTEPS para realizar el análisis de Rasch $^{29}$.

\section{Validación}

Fiabilidad. Fue estimada por el método de consistencia interna y evaluada a través del alfa de Cronbach, aceptando 0,70 como mínimo para comparaciones grupales ${ }^{30}$.

Además se evaluó la correlación ítem-resto del test, a través de la correlación de cada ítem con el resto del instrumento.

Validez de constructo. La estructura multidimensional del instrumento fue evaluada a través del análisis factorial confirmatorio que valora la relación entre los ítems y las dimensiones del instrumento.

La bondad de ajuste se valoró con varios índices: Root Mean Square Error of Approximation (RMSEA) que con valor menor a 0,06 indica ajuste satisfactorio del modelo ${ }^{31,32}$, y con el Comparative Fit Index (CFI) sobre 0,9 l.

Validez discriminante. Mide la capacidad del cuestionario para distinguir entre individuos o poblaciones que se esperan sean diferentes. 
Adaptación y validación del KIDSCREEN-52 para adolescentes chilenos - R. Sepúlveda et al

Tabla 2. Distribución geográfica de la muestra final (n: 7.910)

\begin{tabular}{|c|c|c|c|c|c|}
\hline \multirow[t]{2}{*}{ Región } & \multicolumn{2}{|c|}{ Estudiantes por Región } & \multirow[t]{2}{*}{ Comuna } & \multicolumn{2}{|c|}{ Estudiantes por comuna } \\
\hline & & $\%$ & & n & $\%$ \\
\hline Arica y Parinacota & 291 & 3,7 & Arica & 291 & 3,7 \\
\hline Tarapacá & 314 & 4,0 & Iquique & 314 & 4,0 \\
\hline Antofagasta & 356 & 4,5 & Antofagasta & 356 & 4,5 \\
\hline Atacama & 306 & 3,9 & Copiapó & 306 & 3,9 \\
\hline Coquimbo & 708 & 8,9 & $\begin{array}{l}\text { Coquimbo } \\
\text { La Serena }\end{array}$ & $\begin{array}{l}357 \\
351\end{array}$ & $\begin{array}{l}4,5 \\
4,4\end{array}$ \\
\hline Valparaíso & 1.040 & 13,1 & $\begin{array}{l}\text { Valparaíso } \\
\text { Viña del Mar } \\
\text { San Antonio }\end{array}$ & $\begin{array}{l}397 \\
355 \\
288\end{array}$ & $\begin{array}{l}5,0 \\
4,5 \\
3,6\end{array}$ \\
\hline Maule & 356 & 4,5 & Talca & 356 & 4,5 \\
\hline Bío Bío & 382 & 4,8 & Concepción & 382 & 4,8 \\
\hline De Los Lagos & 397 & 5,0 & Puerto Montt & 397 & 5,0 \\
\hline Magallanes & 395 & 5,0 & $\begin{array}{l}\text { Punta Arenas } \\
\text { Quilicura } \\
\tilde{N} u n ̃ o a\end{array}$ & $\begin{array}{l}395 \\
538 \\
140\end{array}$ & $\begin{array}{l}5,0 \\
6,8 \\
1,8\end{array}$ \\
\hline Región Metropolitana & 3.365 & 42,5 & $\begin{array}{l}\text { Las Condes } \\
\text { Maipú } \\
\text { La Florida } \\
\text { San Bernardo } \\
\text { Santiago }\end{array}$ & $\begin{array}{l}539 \\
444 \\
521 \\
584 \\
599\end{array}$ & $\begin{array}{l}6,8 \\
5,6 \\
6,6 \\
7,4 \\
7,6\end{array}$ \\
\hline Total & 7.910 & 100,0 & & 7.910 & 100,0 \\
\hline
\end{tabular}

Para calcular la validez discriminante se utilizó la comparación de las medias en las cuales otros estudios sobre calidad de vida han reportado diferencias significativas por género y edad ${ }^{33-35}$. Para la validación se utilizó LISREL $8.50^{36}$, STATA 10.0 y SAS 9.1. Para evaluar las diferencias de medias entre hombres y mujeres, entre los rangos de edad y tipo de establecimiento educacional, se utilizó la prueba estadística t de Student y ANOVA con post hoc Duncan.

Este estudio fue aprobado por el Comité de Ética de Investigaciones en seres humanos de la Facultad de Medicina de la Universidad de Chile. La participación fue voluntaria y se solicitó el consentimiento informado de los padres y apoderados y asentimiento informado de los adolescentes. Los datos fueron anónimos y confidenciales. El uso del cuestionario KIDSCREEN, fue respaldado por un convenio entre las Universidades de Chile y Hamburgo de Alemania. El estudio fue financiado por el proyecto Domeyko de la Universidad de Chile y desarrollado entre los años 2008 y 2012.

\section{Resultados}

\section{Adaptación transcultural}

Treinta y uno de los 52 ítems (59,6\%) fueron considerados por las traductoras con bajo nivel de dificultad en la traducción y se logró una alta equivalencia conceptual. En 19 ítems hubo mediano nivel de dificultad en lograr un consenso entre las traductoras y alta equivalencia conceptual. Dos ítems fueron considerados con mayor dificultad para lograr una equivalencia conceptual. A partir de la traducción inversa se cambió un ítem (“Has tenido suficientes oportunidades para salir al aire libre"), en el que se consideró que no había equivalencia conceptual con la versión en inglés original ("Have you had enough opportunity to be outside?").

Las entrevistas cognitivas tuvieron menor duración en el colegio de nivel socioeconómico alto que en el establecimiento de nivel socioeconómico bajo.

Con la información obtenida con las entrevista cognitivas se hizo cambios en once ítems. En cin- 
co de ellos se reemplazó la palabra "padres" por “mamá y/o papá”. La versión final del cuestionario adaptado transculturalmente, tiene 23 ítems que son idénticos a la versión utilizada en España ${ }^{13}$.

\section{Validación}

Fiabilidad: El alfa de Cronbach para el instrumento completo fue de 0,936 y para los dominios puntuaciones mayores a 0,70. Excepción hace: Autopercepción, con 0,62. En todos los ítems el error estándar fue 0,01 (Tabla 3).
Las correlaciones ítem-resto del test, se encontraron entre 0,46 y 0,77 excepto cuatro ítems que se encuentran entre 0,25 y 0,43 .

El análisis factorial confirmatorio mostró que los índices obtenidos para valorar la bondad de ajuste en este estudio fueron de un $\chi^{2}$ $(1.229)=20996,7$, RMSEA (Root Mean Square Error of Approximation $)=0,045$, CFI (Comparative Fit Index $)=0,96$, NFI (Normal Fit Index) $=0,96$, GFI (Goodness of Fit Index) $=0,84$ y RFI (Relative Fit Index $)=0,96$.

Tabla 3. Características psicométricas de las dimensiones e Ítems de la versión chilena del KIDSCREEN-52

\begin{tabular}{|c|c|c|c|c|c|}
\hline \multirow[t]{2}{*}{ Ítem } & \multirow[t]{2}{*}{ Dimensión/Ítem } & \multirow{2}{*}{$\begin{array}{c}\text { Factor de } \\
\text { análisis } \\
\text { confirmatorio } \\
\text { Carga }(\lambda) \\
\left(e^{*} e^{*}\right)\end{array}$} & \multicolumn{2}{|c|}{$\begin{array}{c}\text { Consistencia interna. } \\
\alpha \text { de Cronbach }\end{array}$} & \multirow{2}{*}{$\begin{array}{c}\text { Correlación } \\
\text { Item-Resto } \\
\text { del test }\end{array}$} \\
\hline & & & $\alpha$ & LC $95 \%$ & \\
\hline & Bienestar físico & & 0,75 & $0,74-0,76$ & \\
\hline 1 & En general ¿Cómo dirías que es tu salud? & 0,59 & 0,73 & & 0,48 \\
\hline 2 & ¿Te has sentido bien y en buen estado físico? & 0,74 & 0,69 & & 0,59 \\
\hline 3 & $\begin{array}{l}\text { Has estado físicamente activo/a (ej. Corriendo, esca- } \\
\text { lando, andando en bicicleta, etc.? }\end{array}$ & 0,63 & 0,71 & & 0,52 \\
\hline 4 & ¿Has sido capaz de correr sin dificultad? & 0,63 & 0,71 & & 0,52 \\
\hline \multirow[t]{2}{*}{5} & ¿Te has sentido Ileno/a de energía? & 0,69 & 0,72 & & 0,50 \\
\hline & Bienestar psicológico & & 0,85 & $0,85-0,86$ & \\
\hline 6 & ¿Has disfrutado de la vida? & 0,77 & 0,83 & & 0,67 \\
\hline 7 & ¿Te has sentido contento/a de estar vivo/a? & 0,79 & 0,83 & & 0,65 \\
\hline 8 & ¿Te has sentido satisfecho/a con tu vida? & 0,79 & 0,83 & & 0,65 \\
\hline 9 & ¿Has estado de buen humor? & 0,65 & 0,85 & & 0,56 \\
\hline 10 & ¿Te has sentido alegre? & 0,75 & 0,82 & & 0,68 \\
\hline \multirow[t]{2}{*}{11} & ¿Lo has pasado bien? & 0,72 & 0,83 & & 0,64 \\
\hline & Estado de ánimo y emociones & & 0,85 & $0,84-0,85$ & \\
\hline 12 & ¿Has tenido la sensación de hacerlo todo mal? & 0,69 & 0,83 & & 0,58 \\
\hline 13 & ¿Te has sentido triste? & 0,73 & 0,82 & & 0,63 \\
\hline 14 & ¿Te has sentido tan mal que no querías hacer nada? & 0,77 & 0,82 & & 0,66 \\
\hline 15 & ¿Has sentido en tu vida que todo sale mal? & 0,80 & 0,82 & & 0,70 \\
\hline 16 & $\begin{array}{l}\text { ¿Te has sentido harto/a, (chato/a, choreado/a, } \\
\text { cabreado/a? }\end{array}$ & 0,70 & 0,83 & & 0,63 \\
\hline 17 & ¿Te has sentido solo/a? & 0,69 & 0,83 & & 0,60 \\
\hline \multirow[t]{2}{*}{18} & ¿Te has sentido bajo presión (Presionado/a)? & 0,53 & 0,85 & & 0,46 \\
\hline & Autopercepción & & 0,62 & $0,60-0,63$ & \\
\hline 19 & ¿Has estado contento/a con tu forma de ser? & 0,67 & 0,58 & & 0,37 \\
\hline 20 & ¿Has estado contento/a con la ropa que usas? & 0,52 & 0,59 & & 0,35 \\
\hline 21 & $\begin{array}{l}\text { ¿Has estado preocupado/a por cómo te ves física- } \\
\text { mente? }\end{array}$ & 0,21 & 0,63 & & 0,25 \\
\hline 22 & ¿Has tenido envidia de cómo se ven otros/as niños/as? & 0,52 & 0,55 & & 0,43 \\
\hline 23 & ¿Te gustaría cambiar alguna parte de tu cuerpo? & 0,56 & 0,51 & & 0,50 \\
\hline
\end{tabular}


Tabla 3. Continuación

\begin{tabular}{|c|c|c|c|c|c|}
\hline \multirow[t]{2}{*}{ Ítem } & \multirow[t]{2}{*}{ Dimensión/Ítem } & \multirow{2}{*}{$\begin{array}{c}\text { Factor de } \\
\text { análisis } \\
\text { confirmatorio } \\
\text { Carga }(\lambda) \\
\left(e^{*}\right)\end{array}$} & \multicolumn{2}{|c|}{$\begin{array}{c}\text { Consistencia interna. } \\
\alpha \text { de Cronbach }\end{array}$} & \multirow{2}{*}{$\begin{array}{c}\text { Correlación } \\
\text { Item-Resto } \\
\text { del test }\end{array}$} \\
\hline & & & $\alpha$ & LC 95\% & \\
\hline & Autonomía & & 0,83 & $0,83-0,84$ & \\
\hline 24 & ¿Has tenido suficiente tiempo para ti? & 0,73 & 0,81 & & 0,61 \\
\hline 25 & $\begin{array}{l}\text { ¿Has podido hacer las cosas que has querido en tu } \\
\text { tiempo libre? }\end{array}$ & 0,80 & 0,78 & & 0,69 \\
\hline 26 & $\begin{array}{l}\text { ¿Has tenido suficientes oportunidades para salir al } \\
\text { aire libre? }\end{array}$ & 0,75 & 0,80 & & 0,65 \\
\hline 27 & $\begin{array}{l}\text { ¿Has tenido suficiente tiempo para juntarte con } \\
\text { amigos/as? }\end{array}$ & 0,70 & 0,81 & & 0,60 \\
\hline \multirow[t]{2}{*}{28} & ¿Has podido elegir qué hacer en tu tiempo libre? & 0,71 & 0,81 & & 0,61 \\
\hline & Relación con los padres y vida familiar & & 0,88 & $0,87-0,88$ & \\
\hline 29 & ¿Te han entendido tu mamá y/papá? & 0,79 & 0,85 & & 0,73 \\
\hline 30 & ¿Te has sentido querido/a por tu mamá y/o papá? & 0,83 & 0,86 & & 0,70 \\
\hline 31 & ¿Te has sentido feliz en tu casa? & 0,81 & 0,86 & & 0,69 \\
\hline 32 & $\begin{array}{l}\text { ¿Tu mamá y/o papá han tenido suficiente tiempo } \\
\text { para ti? }\end{array}$ & 0,77 & 0,86 & & 0,70 \\
\hline 33 & ¿Tu mamá y/o papá te han tratado en forma justa? & 0,73 & 0,87 & & 0,63 \\
\hline \multirow[t]{2}{*}{34} & $\begin{array}{l}\text { ¿Has podido hablar mamá y/o papá cuando lo has } \\
\text { querido? }\end{array}$ & 0,74 & 0,86 & & 0,68 \\
\hline & Recursos económicos & & 0,87 & $0,86-0,87$ & \\
\hline 35 & $\begin{array}{l}\text { ¿Has tenido suficiente dinero para hacer las mismas } \\
\text { cosas que tus amigos/as? }\end{array}$ & 0,88 & 0,80 & & 0,77 \\
\hline 36 & ¿Has tenido suficiente dinero para tus gastos personales? & 0,85 & 0,82 & & 0,74 \\
\hline \multirow[t]{2}{*}{37} & $\begin{array}{l}\text { ¿Tienes dinero suficiente para realizar actividades } \\
\text { con tus amigos/as? }\end{array}$ & 0,78 & 0,83 & & 0,74 \\
\hline & Amigos y apoyo social & & 0,81 & $0,80-0,82$ & \\
\hline \multirow[t]{2}{*}{38} & ¿Has pasado tiempo con tus amigos/as? & 0,66 & 0,79 & & 0,58 \\
\hline & ¿Has realizado actividades con otros/as niños/as? & 0,54 & 0,81 & & 0,46 \\
\hline 40 & ¿Lo has pasado bien con tus amigos/as? & 0,81 & 0,78 & & 0,63 \\
\hline 41 & ¿Tú y tus amigos/as se han ayudado entre Uds.? & 0,78 & 0,77 & & 0,64 \\
\hline 42 & ¿Has podido hablar de todo con tus amigos/as? & 0,71 & 0,78 & & 0,60 \\
\hline \multirow[t]{2}{*}{43} & ¿Has podido confiar en tus amigos/as? & 0,65 & 0,79 & & 0,57 \\
\hline & Entorno eEscolar & & 0,80 & $0,79-0,80$ & \\
\hline 44 & ¿Te has sentido feliz en el colegio? & 0,69 & 0,77 & & 0,54 \\
\hline 45 & ¿Te ha ido bien en el colegio? & 0,60 & 0,78 & & 0,49 \\
\hline 46 & ¿Te has sentido satisfecho/a con tus profesores/as? & 0,66 & 0,76 & & 0,58 \\
\hline 47 & ¿Has sido capaz de poner atención? & 0,64 & 0,77 & & 0,55 \\
\hline 48 & ¿Te ha gustado ir al colegio? & 0,68 & 0,76 & & 0,57 \\
\hline \multirow[t]{2}{*}{49} & ¿Te has llevado bien con tus profesores/as? & 0,58 & 0,76 & & 0,57 \\
\hline & Aceptación social & & 0,71 & $0,70-0,73$ & \\
\hline 50 & ¿Has tenido miedo de otros/as niños/as? & 0,73 & 0,69 & & 0,49 \\
\hline 51 & ¿Se han burlado de ti otros /as Niños/as? & 0,61 & 0,60 & & 0,58 \\
\hline 52 & $\begin{array}{l}\text { ¿Te han amenazado o maltratado (física o psicológi- } \\
\text { camente) otros/as niños/as? }\end{array}$ & 0,72 & 0,59 & & 0,56 \\
\hline Total & & & 0,94 & $0,93-0,94$ & \\
\hline
\end{tabular}

Error Standard $=, 01$ en los 52 ítems. 
Adaptación y validación del KIDSCREEN-52 para adolescentes chilenos - R. Sepúlveda et al

Tabla 4. Medias de puntaje Rasch según sexo

\begin{tabular}{|c|c|c|c|}
\hline $\begin{array}{l}\text { Dimensiones } \\
\text { KIDSCREEN-52 }\end{array}$ & $\begin{array}{c}\text { Mujeres } \\
\text { total = } 4.192 \\
\text { Media (DE) }\end{array}$ & $\begin{array}{c}\text { Hombres } \\
\text { total = } 3.718 \\
\text { Media (DE) }\end{array}$ & $\begin{array}{c}\mathbf{t} \\
{ }^{*} p<0,05 \\
{ }^{*} p<0,001\end{array}$ \\
\hline Bienestar físico & $44,9 \quad(10,0)$ & $49,5 \quad(10,6)$ & ** \\
\hline Bienestar psicológico & $49,5 \quad(10,1)$ & $50,9 \quad(9,9)$ & ** \\
\hline Estado de ánimo y emociones & $42,7 \quad(10,6)$ & $45,8 \quad(10,4)$ & ** \\
\hline Autopercepción & $46,5 \quad(8,9)$ & $49,6 \quad(9,1)$ & ** \\
\hline Autonomía & $47,6 \quad(11,7)$ & $49,9 \quad(11,4)$ & ** \\
\hline Relación con los padres y vida familiar & $47,8 \quad(11,7)$ & $49,3 \quad(10,9)$ & ** \\
\hline Recursos económicos & $49,0 \quad(15,1)$ & $49,7 \quad(15,1)$ & * \\
\hline Amigos y apoyo social & $54,2 \quad(11,1)$ & $52,9 \quad(11,2)$ & ** \\
\hline Entorno escolar & $49,4 \quad(9,1)$ & $48,5 \quad(9,1)$ & $* *$ \\
\hline Aceptación social & $54,4 \quad(17,0)$ & $52,2 \quad(16,2)$ & ** \\
\hline
\end{tabular}

Tabla 5. Medias de puntaje Rasch según rango de edad

\begin{tabular}{|c|c|c|c|c|}
\hline Dimensiones KIDSCREEN-52 & $\begin{array}{c}\text { Grupo } 1 \\
10-12 \text { años } \\
\text { total }=2.439 \\
\text { Media (DE) }\end{array}$ & $\begin{array}{c}\text { Grupo } 2 \\
13-15 \text { años } \\
\text { total }=2.948 \\
\text { Media (DE) }\end{array}$ & $\begin{array}{c}\text { Grupo } 3 \\
16-18 \text { años } \\
\text { total }=2.523 \\
\text { Media (DE) }\end{array}$ & $\begin{array}{c}\text { Valor } p \text {, sign. } \\
\text { entre grupos } \\
{ }^{*} \mathbf{F} \\
\mathbf{p}<0,05\end{array}$ \\
\hline Bienestar físico & $49,0 \quad(10,8)$ & $47,0 \quad(10,4)$ & $45,2 \quad(10,3)$ & $1-2 ; 1-3$ y $2-3$ \\
\hline Bienestar psicológico & $52,2 \quad(10,0)$ & $49,5 \quad(10,0)$ & $49,0 \quad(9,9)$ & $1-2$ y $1-3$ \\
\hline Estado de ánimo y emociones & $45,5 \quad(11,0)$ & $43,8 \quad(10,8)$ & $43,2 \quad(9,8)$ & $1-2$ y $1-3$ \\
\hline Autopercepción & $50,2 \quad(10,2)$ & $47,3 \quad(8,6)$ & $46,6 \quad(8,1)$ & $1-2 ; 1-3$ y $2-3$ \\
\hline Autonomía & $50,2 \quad(11,6)$ & $48,8 \quad(11,7)$ & $47,0 \quad(11,3)$ & $1-2 ; 1-3$ y $2-3$ \\
\hline Relación con los padres y vida familiar & $51,0 \quad(11,4)$ & $47,7 \quad(11,3)$ & $47,0 \quad(10,9)$ & $1-2$ y $1-3$ \\
\hline Recursos económicos & $49,1 \quad(15,1)$ & $49,9 \quad(15,1)$ & $48,9 \quad(15,1)$ & - \\
\hline Amigos y apoyo social & $53,4 \quad(11,5)$ & $54,2 \quad(11,2)$ & $53,0 \quad(10,7)$ & $2-3$ \\
\hline Entorno escolar & $51,7 \quad(9,8)$ & $48,3 \quad(8,9)$ & $47,2 \quad(8,1)$ & $1-2 ; 1-3$ y $2-3$ \\
\hline Aceptación social & $49,5 \quad(17,2)$ & $53,5 \quad(16,4)$ & $56,8 \quad(15,6)$ & $1-2 ; 1-3$ y $2-3$ \\
\hline
\end{tabular}

*: ANOVA (F de Fisher-Snedecor, Post hoc: Duncan). Promedio puntaje T (Media de 50 y DE. de 10), utilizando los puntajes Rasch chilenos y estandarización de Europa

En la validez discriminante hubo diferencias significativas entre hombres y mujeres en todas las dimensiones. Las mujeres percibían peor calidad de vida en relación a los hombres en la mayoría de las dimensiones, excepto en "Amigos y apoyo social" y "Aceptación social" (Tabla 4).

Se observan diferencias significativas en las medias obtenidas por grupos de edad (Tabla 5). En la dimensión "Recursos económicos" las diferencias por grupos de edad no fueron significativas.

\section{Discusión}

El KIDSCREEN-52 ha sido el primer instrumento de CVRS para población infantil y adolescente que ha asegurado su equivalencia transcultural, en la experiencia de 13 países europeos. Los resultados presentados permiten la utilización de una versión adecuada culturalmente a Chile y semánticamente equivalente a la versión original.

En las entrevistas cognitivas hubo diferencias 
en el tiempo de respuesta y en la capacidad de comprensión de las preguntas entre los adolescentes de los establecimientos seleccionados (Municipal-Particular) cuyos estudiantes tienen amplias diferencias socioeconómicas. Esto se puede relacionar con la brecha en la calidad de la educación entre los diferentes tipos de colegios en Chile que muestran fuertes diferencias en el Sistema de Medición de Calidad de la Educación (SIMCE), del Ministerio de Educación de Chile, donde se observa que el desempeño de los estudiantes se relaciona con el contexto escolar y social en el que ellos aprenden ${ }^{37}$.

La adaptación chilena se focalizó en población adolescente, pues se aplicó en personas de 10 a 18 años. Originalmente el instrumento es para un rango de 8 a 18 años. En el estudio se hizo una distribución de casi un tercio en cada tipo de establecimiento escolar para no dejar a alumnos fuera del estudio en algunas comunas más pequeñas. A nivel nacional la distribución es de 46,3\% municipal, 46,5\% subvencionada por el Estado y $7,1 \%$ particular pagada ${ }^{26}$. En publicaciones posteriores se ajustarán los análisis por la variable tipo de administración de la educación.

En relación a la bondad de ajuste de los datos a la estructura, estos fueron adecuados al igual que lo fue la versión española ${ }^{13}$. Los resultados obtenidos muestran las buenas propiedades psicométricas encontradas en esta versión chilena del KIDSCREEN-52 que se refleja en los buenos niveles de fiabilidad medido por el Alfa de Cronbach y la correlación ítem vs resto del test. En relación a la validez discriminante, las diferencias encontradas tanto por sexo y grupos de edad, indican que el cuestionario es capaz de discriminar entre grupos en los cuales se esperaba encontrar diferencias según lo publicado por otros estudios ${ }^{33-35,38}$.

El análisis factorial confirmatorio mostró que este instrumento mantiene las dimensiones del test original y que los índices obtenidos para valorar la bondad de ajuste en este estudio fueron muy adecuados ${ }^{31,32}$.

Por lo anterior, podemos concluir que la presente versión chilena del KIDSCREEN-52, tiene una buena fiabilidad y validez para ser aplicado en la población adolescente del país.

Es posible que este cuestionario generado en Europa, no recoja todas las dimensiones relevantes para el estudio de la CVRS en adolescentes chilenos. El estudio Domeyko de la Universidad de Chile, agregó otras 3 dimensiones (Participación Social, Acceso a la Salud, Sexualidad) junto a la aplicación del KIDSCREEN-52, cuyo análisis entregará resultados complementarios, en futuras publicaciones. Posteriores estudios podrían incluir también la versión del cuestionario para padres, considerados como informantes indirectos en los estudios europeos que utilizan el KIDSCREEN.

La adaptación transcultural de este cuestionario lograda para Chile y su validación en una cobertura nacional, permitirá estudios de seguimiento y evaluación de la CVRS en adolescentes escolares.

La versión chilena del KIDSCREEN puede convertirse en un instrumento de gran ayuda en la planificación e intervenciones específicas centradas en la calidad de vida relacionada con la salud de los adolescentes.

\section{Referencias}

1. Centers for Disease Control and Prevention. (2000). Measuring Healthy Days. Atlanta, Georgia: CDC, november 2000. Recuperado el 30 de mayo de 2013, de http://www.cdc.gov/hrqol/pdfs/mhd.pdf

2. Gerharz E, Eiser C, Woodhouse C. Current approaches to assessing the quality of life in children and adolescents. B.J.U International 2003; 91, Suppl 2: 150-4.

3. Urzúa A. Calidad de vida relacionada con la salud: elementos conceptuales. Rev Med Chile 2010; 138 (3): 358-65.

4. Hidalgo-Rasmussen C. Calidad de vida relacionada con la salud en los adolecentes. En: Alonso C, Editor, Salud y enfermedad del Niño y del Adolescente. Jalisco, México: Editorial Manual Moderno; 2013. p. 1560-3.

5. Van Empelen R, Jennekens-Schinkel A, van Rijen PC, Helders PJ, van Nieuwenhuizen $O$. Health related quality of life and self-perceived competence of children assessed before and up to two years after epilepsy surgery. Epilepsia 2005 febrero; 46 (2): 258-71.

6. Lu JC, Cotts TB, Agarwal PP, Attili AK, Dorfman AL. Relation of right ventricular dilatation, age of repair, and restrictive right ventricular physiology with patientreport quality of like in adolescents and adults with repaired tetralogy of fallot. A J Cardiology 2010: 106; (12): 1798-802.

7. Palacio-Vieira JA, Villalonga-Olives E, Alonso J, Valderas JM, Herdman M, Espallargues M, et al. Brief Report: The KIDSCREEN follow-up study on Health-related Quality of Life (HRQoL) in Spanish children and adolescents. 
Pilot test and representativeness. Journal of Adolescence. 2010; 33 (1): 227-31.

8. Ministerio de Salud. Informe final de Encuesta Calidad de Vida y Salud 2006. Julio 2007. Disponible en: http:// epi.minsal.cl/epi/html/sdesalud/calidaddevida2006/ index.htm [Consultado el 18 de agosto de 2012].

9. Urzúa A, Cortés E, Vega S, Prieto L, Tapia K. Propiedades psicométricas del cuestionario de auto reporte de la calidad de vida KIDSCREEN-27 en adolescentes chilenos. Ter Psicol 2009; 27 (1): 83-92.

10. Urzúa A, Cortés E, Prieto L, Vega S, Tapia K. Autoreporte de la calidad de vida en niños y adolescentes escolarizados. Rev Chil Pediatría 2009; 80 (3): 238-44.

11. The World Health Organization Quality of Life assessment (WHOQOL): position Paper from the World Health Organization. Soc Sci Med 1995; 41 (10): 1403 9.

12. Ravens-Sieberer U, Gosch A, Rajmil L, Erhart M, Bruil J, Duer W, et al. KIDSCREEN Group E. KIDSCREEN-52 quality-of-life measure for children and adolescents. Expert Rev of Pharmacoecon Outcomes Res 2005; 5 (3): 353-64.

13. Aymerich M, Berra S, Guillamon I, Herdman M, Alonso J, Ravens- Sieberer U, et al. Desarrollo de la versión en español del KIDSCREEN, un cuestionario de calidad de vida para la población infantil y adolescente. Gac Sanit 2005; 19 (2): 93-102.

14. Ravens-Sieberer U, Gosch A, Rajmil L, Erhart M, Bruil J, Power M, Duer W, et al. KIDSCREEN Group. THE KIDSCREEN-52 Quality of life measure for children and adolescents: psychometric results from a cross-cultural survey in 13 European countries. Value Health 2008; 11 (4): 645-58.

15. Tebe C, Berra S, Herdmanb M, Aymerich M, Alonso J, Rajmil L. Fiabilidad y validez de la versión española del KIDSCREEN-52 para población infantil y adolescente Med Clin (Barc) 2008; 130 (17): 650-4.

16. Berra S, Bustingorry V, Henze C, Díaz M del P, Rajmil L, Butinof M. Adaptación transcultural del cuestionario KIDSCREEN para medir calidad de vida relacionada con la salud en población argentina de 8 a 18 años. Arch Argent Pediatr 2009; 107 (4): 307-14.

17. Jaimes-Valencia M, Richard M, Cabrero J, FloresAlarcón L, Palacio J. Calidad de vida relacionada con la salud en niños(as)-adolescentes: adaptación cultural del instrumento KIDSCREEN-52 en Colombia. En Libro X Encuentro Internacional de Investigación en Enfermería, Albacete, España. Eds. Instituto de Salud Carlos III y Unidad de coordinación y desarrollo de la Investigación en Enfermería, Madrid; 2006. p. 75-7. Disponible en: http://www.google.cl/url?sa $=t \& r c t=j \& q$
$=\&$ esr $\mathrm{c}=\mathrm{s} \&$ source $=$ web $\& \mathrm{~cd}=1 \& \mathrm{ved}=0$ CFAQFjAA\&url $=$ http $\% 3 \mathrm{~A} \% 2 \mathrm{~F} \% 2 \mathrm{Fwww}$. isciii.es $\% 2 \mathrm{FISCIII} \% 2 \mathrm{Fes} \% 2 \mathrm{FcO}$ ntenidos\%2Ffd-el-instituto\%2Ffd-organizacion $\% 2 \mathrm{Ffd}-$ estructura-directiva $\% 2 \mathrm{Ffd}$-subdireccion-general-redescentros-investigacion $2 \% 2 \mathrm{Ffd}$-centros-unidades $2 \% 2 \mathrm{Ffd}$ investen-isciii-2\%2Fdocus\%2F2006_X_encuentro_Investen_Albacete.pdf\&ei=zX0MUKr3H4m69gSBidX4C g\&usg=AFQjCNF2Kw2vRcFp3L2yflgvOqW59GuTgw. Consultado el 18 de agosto de 2012.

18. Guzmán de RN, Richart M. Transcultural Adaptation and Validity Of KIDSCREEN and DISABKIDS Questionnaires For Children And Teenagers In Venezuela. 2008 International Society for Quality of Life Research (meeting abstracts $145515^{\circ}$ Annual Conference of ISOQOL, Montevideo, Uruguay) The QLR Journal 2008; A 31, Abstract \# 1455. Disponible en: www.isoqol.org/pdfs/ AbstractsForBooklet_2008v3.pdf. [Consultado el 18 de agosto de 2012].

19. Scientific Advisory Comitee of the Medical Outcomes Trust. Assessing health status and quality-of-life instruments: Attributes and review criteria. Quality of Life Research. 2002; 11: p. 193-205.

20. The KIDSCREEN Group. Kidscreen. [Online]. 2004 [cited 2009 noviembre 17. Available from: http://kidscreen. port4949.net/cms/sites/kidscreen.port4949.net/files/ KIDSCREEN_Translation_Manual.pdf.

21. Beaton De, Bombardier C, Guillemin F, Ferraz MB. Guidelines for the process of cross-cultural adaptation of self-report measures. Spine 2000; 25 (24): 3186-91.

22. Schmidt $S$, Bullinger M. Current issues in cross-cultural quality of life instrument development. Arch Phys Med Rehabil 2003; 84 (Suppl 2): S29-34.

23. Wild D, Grove A, Martin M, Eremenco S, McElroy S, Verjee-Lorenz A, et al. Principles of good practice for the translation and cultural adaptation process for patientreported outcomes (PRO) measures: report of the ISPOR Task Force for translation and cultural adaptation. Value Health 2005; 8 (2), 94-104.

24. The KIDSCREEN Group. Existing language versions, 2010. http://www.kidscreen.org/cms/existinglanguageversions. Consultado el 29 de julio de 2010.

25. Chen Won Sun. Questionnaire translation and psychometric properties evaluation. 2004 Disponible en: http:// www.onlinereview.segi.edu.my/chapters/vol2_chap5.pdf. Consultado el 18 de agosto de 2012.

26. Ministerio de Educación de Chile. Departamento de Estudios y Desarrollo de la División de Planificación y Presupuesto. Indicadores de la Educación en Chile 2007-2008. Disponible en: http://w3app.mineduc.cl/ mineduc/ded/documentos/indicadores_2007-2008.pdf [Consultado el 18 de agosto de 2012]. 
27. Ministerio de Salud de Chile. Encuesta de Calidad de Vida 2006. Disponible en: http://epi.minsal.cl/epi/html/ sdesalud/calidaddevida2006/Informe\%20Regional\%20 ENCAVI\%202006.pdf. [Consultado el 18 de agosto de 2012].

28. Vélez Gálarra R, López Águila S, Rajmil L. Género y salud percibida en la infancia y la adolescencia en España. Gac Sanitaria 2009; 23 (5): 433-9.

29. Linacre J. A user guide to winsteps. Rasch model computer program. Chicago, IL: MESA Press. 2004.

30. The Netherlands Cancer Institute, Amsterdam. Trust Assessing health status and quality-of-life instruments: Attributes and review criteria. Quality of life Research 2002; 11 (3): 193-205.

31. Bentler PM, Bonett DG. "Significance Tests and Goodness of Fit in the Analysis of Covariance Structures". Psychological Bulletin 1980; 88: 588-606.

32. Bollen KA. Structural equations with latent variables. New York, NY: John Wiley \& Sons. 1989.

33. Serra-Sutton V, Rajmil L, Alonso J, Riley A, Starfield B. Valores poblacionales de referencia del perfil de salud CHIPAE a partir de una muestra representativa de ado- lescentes escolarizados. Gac Sanit 2003; 17 (3): 181-9.

34. Bisegger C, Cloetta B, von Rueden U, Abel T, RavensSieberer U. European Kidscreen Group. Health-related quality of life: gender differences in childhood and adolescence. Soz Praventivmed 2005; 50 (5): 281-91.

35. Ravens-Sieberer U, Gosch A, Rajmil L, Erhart M, Bruil J, Duer W, et al. European KIDSCREEN Group KIDSCREEN-52 quality of life measure for children and adolescents Expert Rev Pharmacoecon Outcomes Res 2005; 5 (3): 353-64.

36. Joreskog KG, Van Thillo M. "LISREL: A General Computer Program for Estimating a Linear Structural Equation System Involving Multiple Indicators of Unmeasured Variables (RB-72-56)". Princeton, NJ: Educational Testing Service 1972. Disponible en: http://www.ssicentral. com/lisrel/ [Consultado el 18 de agosto de 2012].

37. Sistema de Medición de Calidad de la Educación (SIMCE). Disponible en: www.simce.cl [Consultado el 18 de agosto de 2012].

38. García X, Pérez A, Adell M. Factores relacionados con el acoso escolar (bullying) en los adolescentes de Barcelona. Gac Sanit 2010; 24 (2): 103-8. 